\title{
VIRTUAL REALITY IN PARAPLEGIA: A TEST BED APPLICATION
}

\author{
Giuseppe Riva \\ Istituto Auxologico Italiano, IRCCS \\ Verbania, Italy
}

\begin{abstract}
The paper presents an overview of the ergonomic/design issues of a VR-enhanced orthopaedic appliance to be used in rehabilitation of patients with Spinal Cord Injury. First, some design considerations are described and an outline of aims which the tool should pursue are given. Finally, the design issues are described focusing both on the development of a test-bed rehabilitation device and on the description of a preliminary study detailing the use of the device with a long-term SCI patient. The basis for this approach is that physical therapy and motivation are crucial for maintaining flexibility and muscle strength and for reorganizing the nervous system after SCIs.
\end{abstract}

\section{Introduction}

The design of rehabilitation equipment is primariy human-centred and relies on the understanding of the users needs as well as on the manner in which his/her disability is anticipated evolve in time [14]. So to develop a good rehabilitation device, a comprehension of all aspects of the rehabilitation process is required.

The main purpose of rehabilitation after a spinal cord injury (SCI) is to help the patient establish control over his/her own health and life [6]. Aspects of rehabilitation include:

- regaining as much strength and function in the trunk and extremities as possible [27];

- management of bladder and bowel function $[3,17]$;

- independent personal and community mobility $[11,18,30]$;

- $\quad$ psychosocial and sexual function $[1,9,26]$;

- $\quad$ and independent living and vocational rehabilitation [7];

Motivation and self-confidence, are also major factors in successful rehabilitation [12, 16, 28]. They are required, first, to accept irreversible facts. It takes still more motivation and self-esteem to make the most of what have not been injured - talent, creative energies, intellectual resources.

A common problem in SCIs rehabilitation is the failure to address the pathophysiological response to trauma: specific problem areas such as memory or activities of daily living cannot be pursued without adequate attention to the likely contribution from underlying neuropathophysiological factors [15]. The rehabilitation process, for example, may not produce improvements in the specific areas targeted, but benefit may arise in an improved general state of arousal-activation or motivation of individual recipients. 
In this sense, it is reasonable to suggest that a logical first step in any rehabilitation programme should be to facilitate this fundamental process $[15,29]$. A more activated brain may be better able to benefit from more traditional rehabilitation interventions. Moreover, it could be useful to treat a common motor problem, muscle incoordination, at least partially induced by the substantial brain reorganization that occurs after injury to the central nervous system.

Actually, an accessible route to improve arousal-activation may be by way of physical exercise, which has unequivocal physical and mental benefits. In fact, inactivity accelerates the rates of decline of major physiological adaptive systems which eventually reach the point at which the individual's ability to prevent or recover from acute stresses is impaired. The individual's ability to cope with such stresses and preserve subsequent function depends upon the maintenance of adequate physiological reserves, particularly neurological control, mechanical performance, and energy metabolism. Activity and exercise are increasingly recognised as a way of improving mental health, with reports of reductions in depression and anxiety, and increased perceptions of self control [15]. The most likely basis for these benefits is a change in central monoaminergic activity. For example, it is suggested that the antidepressant effect of physical exercise shifts monoaminergic function back to normal in clinically depressed subjects.

Physical rehabilitation therapy can also help to restore depressed immune system functionality [8]. It is well known that in SCI patients both natural and adaptive immune responses decrease strikingly within 3 months after injury. However, Cruse et al. [8] reported that physical therapy was able to restore immune resposes to near normal levels in most patients of the tested sample. Natural immune system depression persisted in spinal cord injury patients not receiving physical rehabilitation therapy.

Finally, results from animal experimental studies suggest that chronic exercise may also result in permanent structural changes in the brain [15]. For instance, Black et al. [4], found that physical exercise in rats improves vascularisation in the cerebellar cortex, while a combination of motor learning with physical activity results in a greater communication network within the brain. Moreover, recent studies in animals with spinal cord injuries indicated that physical therapy can also have a key role in recovery after SCI. In fact, data showed that recovery of movement is linked to the types of training the animals receive; physical therapy and other rehabilitation strategies are crucial for maintaining flexibility and muscle strength and for reorganizing the nervous system.

Although there is much potential for the use of immersive virtual reality environments in clinical rehabilitation, some problems have limited their application in this field. Some users have experienced side-effects,during and after exposure to immersive virtual reality environments. The symptoms experienced by these users are similar to those which have been reported during and after exposures to simulators with wide field-of-view displays [20]. These side-effects have been collectively referred to as "simulator sickness" and are characterised by three classes of symptoms: ocular problems, such as eyestrain, blurred vision, and fatigue; disorientation and balance disturbances; and nausea [25]. While in some applications cybersickness is reported to be negligible [23], exposure duration of less than 10 minutes in some immersive virtual reality environments has been reported to result in significant incidences of nausea, disorientation, and ocular problems [19]. 


\section{Virtual Reality in SCI Rehabilitation}

Given these premises, physical therapy is usually a major part of the treatment program in rehabilitation centers [5]. Usually a full physical therapy program for SCI patients includes:

- Progressive resistive exercises. These are exercises done with weights, pulleys, and special exercise machines.

- Mat class. Working on a mat, the patient relearns and practices the skills needed for independent living: changing position in bed, getting dressed, and moving from one place to another.

- Tilt table. A table that can be positioned at various angles to the horizontal helps the cardiovascular system readjust to upright position after a patient has been in bed for extended periods.

- Wheelchair class. The patient learns to handle a wheelchair, especially on curbs, ramps, stairs, and in a car.

However, long-term physical therapy is very demanding and requires a strong motivation in patients. A strong possibility for overcoming these problems could be obtained from Virtual Reality (VR). The basis for a "VR approach" is that a computer can synthesise a three-dimensional graphical environment from numerical data. Using visual and auditory output devices - usually head mounted displays - the human operator can experience the environment as if it were a part of the world. Further, because input devices sense the operator's reactions and motions, the operator can modify the synthetic environment, creating the illusion of interacting with, and thus being immersed within, the environment.

VR is highly flexible and programmable. It enables one to present a wide variety of controlled stimuli and to measure and monitor a wide variety of responses by the user [22, 24]. Both the synthetic environment itself and the manner in which this environment is modified by the user's responses can be tailored to the needs of each client and/or therapeutic application [13]. Moreover, VR is highly immersive and can cause the participant to feel "present" in the virtual rather than the real environment. In this sense VR provides a powerful means of increasing levels of environmental interaction in a highly controlled and structured manner. In fact, as noted by Johnson et al. [15], a VR enriched environment offers the potential for significant gains in physical and mental function at all levels. This is especially true if patients are introduced to a graded physical exercise programme within an enriched VR environment with the aim of increasing physical and mental parameters.

To reach this goal we have developed a test-bed orthopaedic appliance for walking and rehabilitation comprising:

- a semi-rigid exoskeleton for support of the bust and the lower limbs;

- a virtual reality system.

The device was developed according to the guidelines detailed previously and is designed to be easy tailored to the needs of different users (Figure 1). The test-bed application was designed to reproduce the feeling of an excursion to the mountains. The expected end-user cost of the appliance, including the adaptation process required to match the specific physical characteristics of each SCI patient, is about $\$ 15,000-20,000$. A more detailed description of the apparatus will now be given, together with the description of a preliminary case report detailing the use of the device with a long-term SCI patient. 

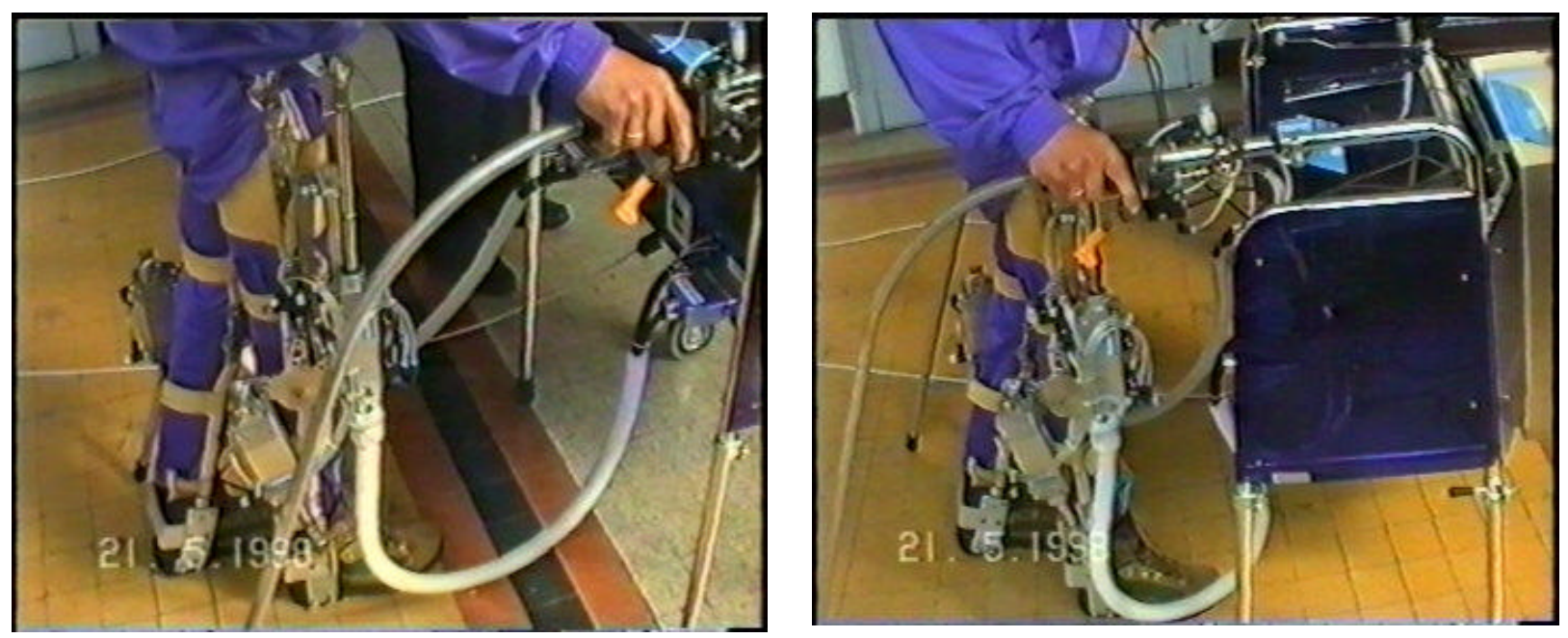

Figure 1: The test-bed application

\section{System Design and Implementation}

The gait-inducing exoskeleton, patented worldwide, was designed and developed by Ferrati Benito, President and CEO of Ferrati Electronic, an Italian company based near Milano. It consists of a compressed air operated semi-rigid sling for supporting the bust and the lower limbs of the patient, together with a framework designed as stable rest and support for the user.

The sling is equipped with small actuators (microcylinders) activated by compressed air that move the lower jointed part of the sling in accordance with the human gait. The microcylinders are operated by the patient by means of a two-button interface located on both sides of the framework grips: pressing the left button causes the left leg to move forward; pressing the button on the right causes the right leg to move forward. The framework has grips on both sides, wheels are there for moving on the floor, and inside are located both the PC used for the virtual experience and the compressed-air delivery system.

The VR-enhanced orthopaedical appliance uses a Thunder 400/C virtual reality system by Virtual Engineering of Milano-Italy. The Thunder 400/C is a Pentium II based immersive VR system (400mhz, 32 mega RAM, Matrox Millenium II 8Mb Wram graphic engine) including an HMD subsystem.

A head mounted display (HMD) with $40^{\circ} \mathrm{H}$ and $30^{\circ} \mathrm{V}$ field of view (50 diagonal) provides the visual display. The HMD, developed by Retinal Displays Inc of Los Altos (CA) for Virtuality (UK), displays 800 lines of 225 pixels (180,000 active dots) to each eye and uses LCD technology (a full color AMLCD panel). The provided head tracker is used to sense head rotation.

The HMD does not have a stereoscopic display. Previous researches regard stereoscopy as important because it provides the user with good cues of depth [2]. However, the refresh rate of graphics decrease by $50 \%$ when a different image is presented to each eye, as required by a stereoscopic display. Consequently, we decided against implementing a stereoscopic display. To compensate for the lack of binocular cues, we included perspective cues (light and shade, relative size, textural gradient, interposition and motion parallax) in the virtual environment [10]. 
The virtual environment used in the study was developed by Virtual Engineering, a company based in Milano, Italy, using VRT 5.5 from Superscape Ltd. (UK). The environment is designed to reproduce the feeling of an excursion to the mountains, by simulating a stroll through a mountain path. To increase the realism of the experience, actual images of Alpine scenery were used together with sounds and voices typical of a natural environment. The virtual environment moves in sync with the patient's steps: each motion ahead is activated by the buttons located on both side of the framework.

\section{A Preliminary Study}

The first VR-enhanced orthopaedical appliance was developed for Nicola, a 26-year old patient with complete paraplegia. Two experimental sessions were conducted. Each session was composed by two fifteen-minute system trials separated by a ten-minute rest period. During each trial, Nicola completed the experience by walking through the virtual path till the peak of a snow-covered mountain Some sample images along that path are shown in Figure 2. During the second trial, a virtual runner was added and the patient had to compete with him for reaching first the peak of the mountain. The two images of Figure 3 illustrate.
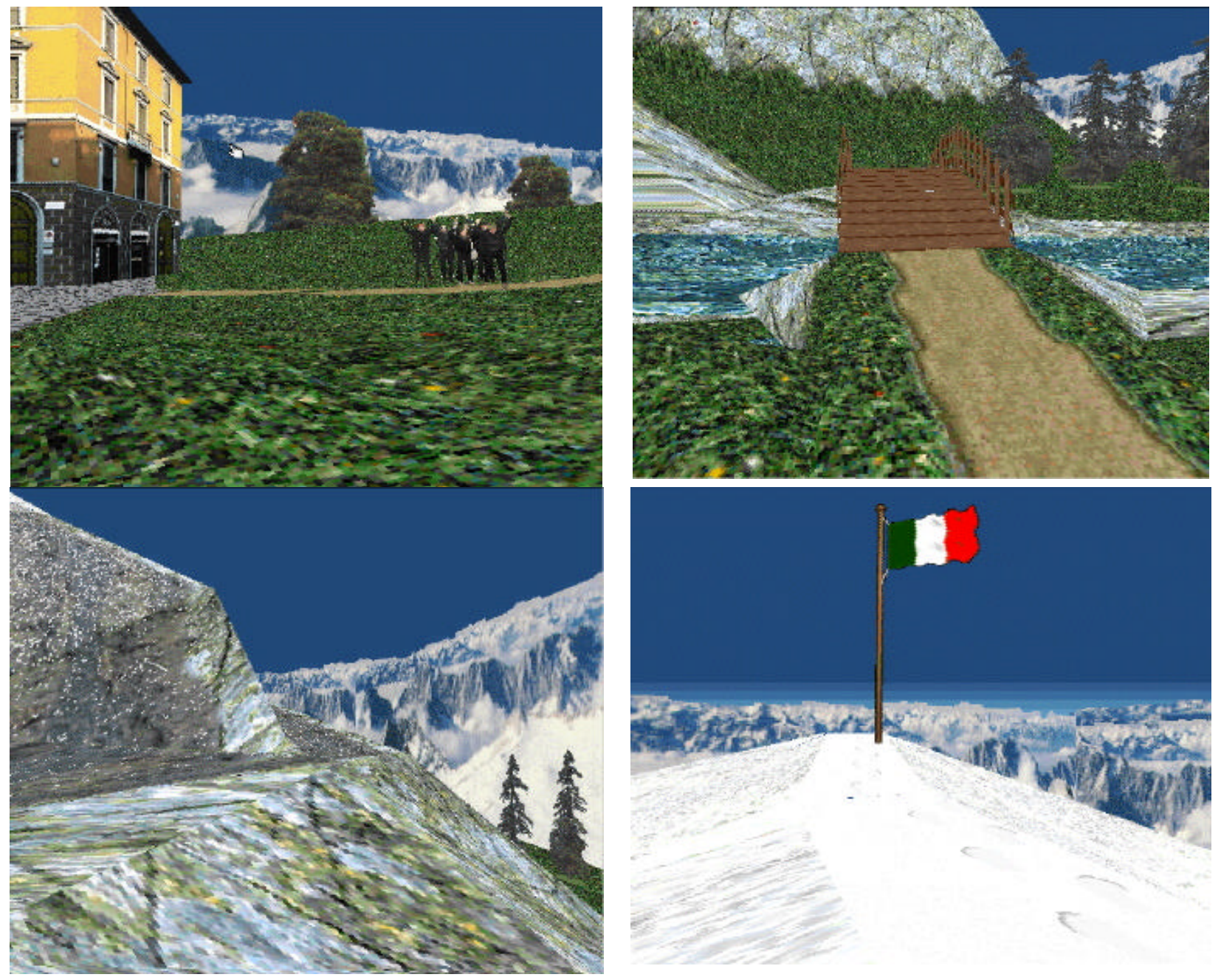

Figure 2: Scenes along the virtual path 

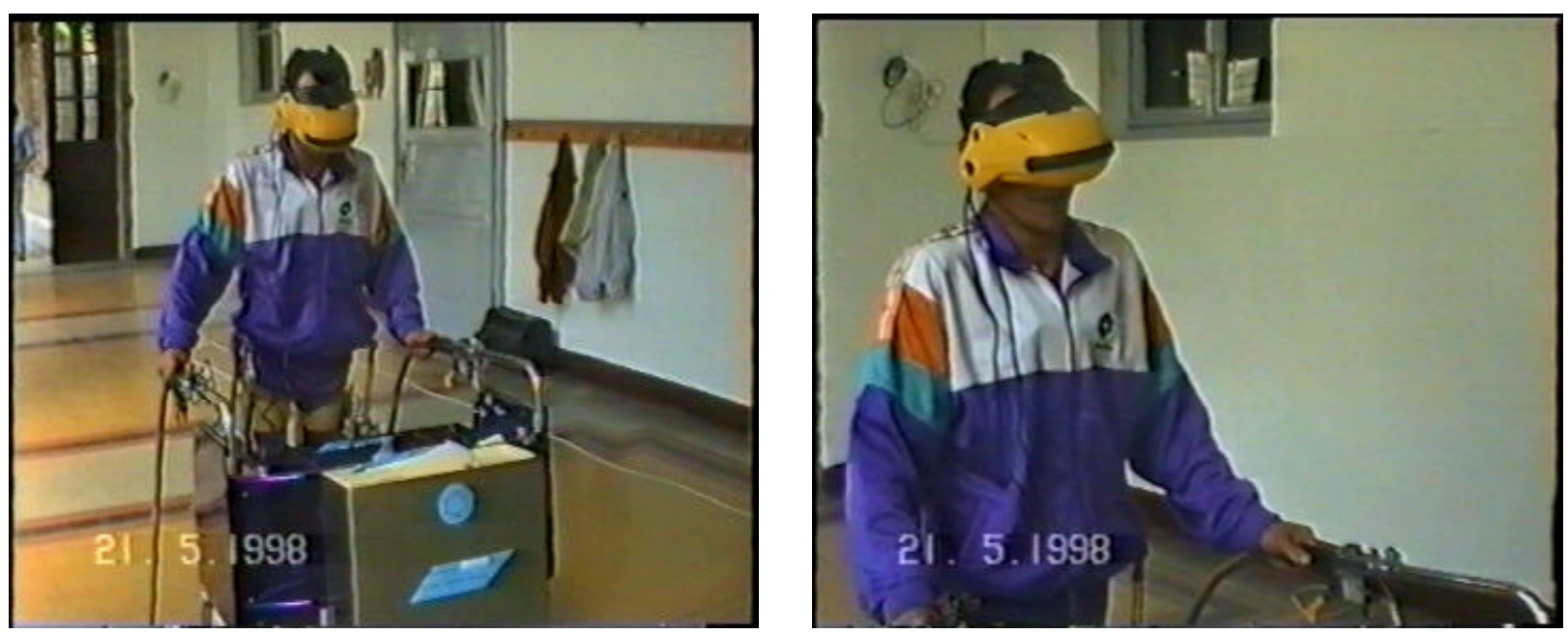

Figure 3: Patient competing with virtual runner

Before and after each session Nicola had to assess his emotional and physical state using a questionnaire constructed according to the model of the semantic differential ratings [21]. The questionnaire was composed by 20 bipolar adjectives, each item having six levels of intensity (Table 1).

\begin{tabular}{|c|c|c|c|c|c|c|c|}
\hline \hline & 1 & 2 & 3 & 4 & 5 & 6 & \\
\hline Fast & & & & & & & Slow \\
\hline Unpleasant & & & & & & & Pleasant \\
\hline Secure & & & & & & & Insecure \\
\hline Unhappy & & & & & & & Happy \\
\hline Irvariable & & & & & & & Variable \\
\hline Beautiful & & & & & & & Nasty \\
\hline Weak & & & & & & & Strong \\
\hline Heavy & & & & & & & Light \\
\hline Optimistic & & & & & & & Pessiruistic \\
\hline Capable & & & & & & & Irrapable \\
\hline Active & & & & & & & Passive \\
\hline Good & & & & & & & Bad \\
\hline Tense & & & & & & & Relaxed \\
\hline Indolent & & & & & & & Willing \\
\hline Likeable & & & & & & & Unlikeable \\
\hline Dinaric & & & & & & & Static \\
\hline Imprudent & & & & & & & Prudent \\
\hline Indispensable & & & & & & & Superfluous \\
\hline Warm & & & & & & & Cold \\
\hline Brave & & & & & & & Fearful \\
\hline
\end{tabular}

Table 1: Scoring Sheet For Semantic Differential Ratings 
The first result of this study is the lack of side effects and simulation sickness in our patient after both session, suggesting the possibility of using VR for therapeutic purposes. Only during the first session Nicola reported an increased level of fatigue together with a slight pain in the left ankle.

From the analysis of the obtained ratings we found: a slightly improved level of in self-confidence (Before first session: 2; after second session: 3); higher level of optimism (Before first session: 4; after second session: 6) and will (Before first session: 4; after second session: 5); increased relaxation (Before first session: 1; after second session: 3) and activity (Before first session: 1; after second session: 2) scores. In general the highest scores were obtained after the end of the second session.

Nicola also declared subjective improvement in his sense of well-being, mood and quality of sleep. The improvement in the sense of well-being can be also verified by higher scores both in mood-related adjectives (likeable-unlikeable and happy-unhappy) and in state-related adjectives (fast-slow and dynamic-static).

\section{Conclusions}

While in some applications, cybersickness is reported to be negligible [23], exposure duration of less than 10 minutes in some immersive virtual reality environments has been reported to result in significant incidences of nausea, disorientation and ocular problems [19]. The first result of this study is the lack of side effects and simulation sickness in our patient after both session, confirming the possibility of using VR for therapeutic purposes.

Moreover, this type of VR system may offer some advantages over currently used rehabilitation devices:

- the potential to increase the motivation of the patient. As we have seen before, the overall adjustment to a new life style is the overwhelming problem for the paraplegic. As one paralyzed veteran put it: "The impact on the patient is traumatic, to say the least. There is the realization that a once whole and healthy body is no longer fully functional and is plagued with a myriad of secondary disabilities. It requires motivation, first, to accept irreversible facts. It takes still more motivation to make the most of what has not been injured - talent, creative energies, intellectual resources. The possibility for SCI patients of experimenting again the walking experience, even if wearing an exoskeleton and only for a few minutes, could have positive effects on motivation and emotional status.

- the capability of developing an enriched environment which offers the potential for significant gains in physical and mental function at many levels. As we have seen before, physical therapy is able to to improve arousal-activation, to treat muscle incoordination and to restore immune resposes. Moreover, the use an enriched virtual environment could help to improve the effects of new drug therapies (e.g. methylprednisolone or GM-1 gangliosideor) or neural prostheses. In fact, as noted by NINDS researcher, Physical therapy and other rehabilitation strategies are crucial for maintaining flexibility and muscle strength and for reorganizing the nervous system. These factors could be vital to recovering movement following regeneration as well as maximizing the use of undamaged nerve fibers. 
Of course these results are preliminary. From a clinical view point the issues that we have to address in the future are: further testing of the device using controlled clinical trials; a follow-up study to check the persistence of the obtained results and how to integrate the use of the orthopaedical appliance into the standard rehabilitation practice.

\section{REFERENCES}

[1] C. J. Alexander, Psychological assessment and treatment of sexual dysfunctions following spinal cord injury, J Am Paraplegia Soc, vol. 14, pp. 127-31, 1991.

[2] P. T. Barham and D. F. Mc Allister, A comparison of stereoscopic cursors for the interactive manipulation of B-splines, presented at Stereoscopic Display and Applications II, 1991.

[3] L. S. Baskin and R. A. Schmidt, Bladder rehabilitation with dorsal rhizotomy and ventral neuroprosthesis, Paraplegia, vol. 30, pp. 783-7, 1992.

[4] J. E. Black, K. R. Isaacs, and B. J. Anderson, Learning causes synaptogenesis, whereas motor activity causes angiogenesis, in cerebellar cortex of adult rats, Proceedings of the National Academy of Sciences - USA, vol. 87, pp. 5568-5572, 1990.

[5] M. J. Buck, [Integration of patient exercise/physical therapy in the rehabilitation team], Rehabilitation, vol. 31, pp. 154-6, 1992.

[6] M. F. Cawley, G. M. Yarkony, and S. B. Bergman, Spinal cord injury rehabilitation. 5. Through the lifespan, Arch Phys Med Rehabil, vol. 78, pp. S73-8, 1997.

[7] R. Crisp, Vocational decision making by sixty spinal cord injury patients, Paraplegia, vol. 30, pp. 420-4, 1992.

[8] J. M. Cruse, R. E. Lewis, G. R. Bishop, W. F. Kliesch, and E. Gaitan, Neuroendocrine-immune interactions associated with loss and restoration of immune system function in spinal cord injury and stroke patients, Immunology Research, vol. 11, pp. 104-16, 1992.

[9] M. L. Curcoll, Psychological approach to the rehabilitation of the spinal cord injured: the contribution of relaxation techniques, Paraplegia, vol. 30, pp. 425-7, 1992.

[10] Q. E. Dolecek, Computer-generated stereoscopic displays, John Hopkins APL Technical Digest, vol. 15, pp. 137-142, 1994.

[11] F. Felici, M. Bernardi, A. Radio, P. Marchettoni, V. Castellano, and A. Macaluso, Rehabilitation of walking for paraplegic patients by means of a treadmill, Spinal Cord, vol. 35, pp. 383-5, 1997.

[12] D. M. Furst, T. Ferr, and N. Megginson, Motivation of disabled athletes to participate in triathlons, Psychol Rep, vol. 72, pp. 403-6, 1993. 
[13] K. Glantz, N. I. Durlach, R. C. Barnett, and W. A. Aviles, Virtual reality (VR) for psychotherapy: From the physical to the social environment, Psychotherapy, vol. 33, pp. 464-473, 1996.

[14] J. Ibañez-Guzmán, Design of rehabilitation robots using virtual work platforms, presented at First European Conference on Disability, Virtual Reality and Associated Technology, Maidenhead, UK, 1996.

[15] D. A. Johnson, S. Rushton, and J. Shaw, Virtual reality enriched environments, physical exercise and neuropsychological rehabilitation, presented at First European Conference on Disability, Virtual Reality and Associated Technology, Maidenhead, UK, 1996.

[16] S. A. Jordan, W. R. d. Wellborn, J. Kovnick, and R. Saltzstein, Understanding and treating motivation difficulties in ventilator- dependent SCI patients, Paraplegia, vol. 29, pp. 431-42, 1991.

[17] K. C. Mudgal, R. Gupta, S. Singh, J. S. Bhatia, and M. Kalra, Bladder rehabilitation in spinal cord injury patients, J Indian Med Assoc, vol. 90, pp. 52-4, 1992.

[18] R. G. Platts and M. H. Fraser, Assistive technology in the rehabilitation of patients with high spinal cord lesions, Paraplegia, vol. 31, pp. 280-7, 1993.

[19] E. C. Regan and K. R. Price, Some side-effects of immersion virtual reality: the effects of increasing head movements, of rapid interaction, and of seated subjects, Army Personnel Research Establishment, Farnborough (UK) Report 93R022, 1993.

[20] E. C. Regan and K. R. Price, The frequency of occurrence and severity of side-effects of immersion virtual reality, Aviat Space Environ Med, vol. 65, pp. 527-30, 1994.

[21] G. Riva, The role of emotional and socio-cognitive patterns in obesity: Eating attitudes in obese adolescents before and after a dietary-behavioral therapy, Psychological Reports, vol. 79, pp. 3546, 1996.

[22] G. Riva, Virtual reality as assessment tool in psychology, in Virtual reality in neuro-psychophysiology, G. Riva, Ed. Amsterdam: IOS Press, 1997, pp. 95-112.

[23] G. Riva, Virtual environment for body-image modification: Virtual reality system for the treatment of body image disturbances, Computers in Human Behavior, vol. 14, pp. 477-490, 1998.

[24] G. Riva, Virtual reality in psychological assessment: The Body Image Virtual Reality Scale, CyberPsychology \& Behavior, vol. 1, pp. 37-44, 1998.

[25] G. Riva, Virtual Reality vs. Virtual Body: The use of virtual environments in the treatment of body experience disturbances, CyberPsychology \& Behavior, vol. 1, pp. 129-137, 1998.

[26] M. L. Sipski, The impact of spinal cord injury on female sexuality, menstruation and pregnancy: a review of the literature, J Am Paraplegia Soc, vol. 14, pp. 122-6, 1991.

[27] K. E. Sloan, L. A. Bremner, J. Byrne, R. E. Day, and E. R. Scull, Musculoskeletal effects of an electrical stimulation induced cycling programme in the spinal injured, Paraplegia, vol. 32, pp. 407-15, 1994. 
[28] M. Solomonow, E. Aguilar, E. Reisin, R. V. Baratta, R. Best, T. Coetzee, and R. Dambrosia, Reciprocating gait orthosis powered with electrical muscle stimulation (RGO II). Part I: Performance evaluation of 70 paraplegic patients, Orthopedics, vol. 20, pp. 315-24, 1997.

[29] J. P. Wann and J. D. Turnbull, Motor skill learning in cerebral palsy: movement, action and computer- enhanced therapy, Baillieres Clin Neurol, vol. 2, pp. 15-28, 1993.

[30] G. Wheeler, K. Krausher, C. Cumming, V. Jung, R. Steadward, and D. Cumming, Personal styles and ways of coping in individuals who use wheelchairs, Spinal Cord, vol. 34, pp. 351-7, 1996.

\section{BIOGRAPHY}

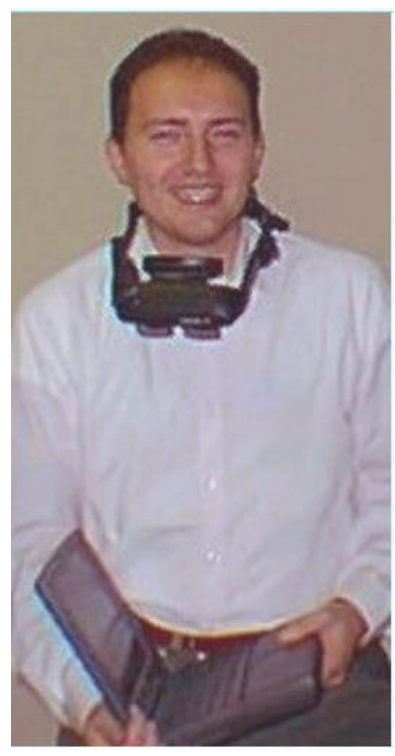

Giuseppe Riva, Ph.D., is Senior Researcher at the Applied Technology for Neuro-Psychology Laboratory - ATN-P Lab., Istituto Auxologico Italiano, Milan, Italy, and Professor of Applied Social Psychology (Psicologia degli Atteggiamenti e delle Opinioni) at the Cagliari State University, Sardinia, Italy. He received his Ph.D in Social and Developmental Psychology from the Catholic University of Milan, Italy. In the ATN-P Lab. he conducted several researches and published many papers about methods and assessment tools in psychology and about the use of Virtual Reality in medicine and in training. He has also played a key role in several researches carried out by the GRICO, Gruppo di Ricerca sull'Interazione COmunicativa (Research Group on Communicative Interaction) of the Catholic University of Milan, many of which focused on issues concerning methodological and psychological implicancies in the use of new information technologies. A member of the New York Academy of Science, Riva is actually Associate Editor for the "CyberPsychology \& Behavior" journal.

\section{Contact Information:}

Applied Technology for Neuro-Psychology Lab.

Istituto Auxologico Italiano, IRCCS

Verbania, Italy

P.O. Box 1

28044 Verbania - Italy

Phone: +39 - 323 - 580278

Fax: +39 - 323 - 587694

Email link: mailto:auxo.psylab@auxologico.inet.it

Web: http://www.psicologia.net/ and http://www.ehto.be/ht projects/vrepar 